\title{
A Future Full of Variety
}

Dear Reader,

The automotive industry is faced with major disruptive changes. The key questions that we have to answer include: What mobility concepts do we need in order to meet the requirements of tomorrow? How should we structure the energy supply chain for transport in order to guarantee that future mobility can operate effectively? The main drivers are climate change, urbanization, digitization, and automation. At the $11^{\text {th }}$ Schaeffler Colloquium in Baden-Baden, Schaeffler CTO Prof. Peter Gutzmer emphasized:

"There is no such thing as a car-friendly city, we need to find other solutions. Levels of intermodal transport will increase significantly. How can we develop more efficient transport services?" Europe is lagging behind in this area. In other parts of the world, there is a much greater focus on intelligent and connected mobility solutions, on zero local emissions, and on the use of renewable energy in the urban planning process. Another important consideration is reducing the volume of traffic by introducing individualized, pedestrian-focused mobility solutions, which include robo-taxis, pedelecs, and cars like the Schaeffler bio-hybrid.

In the process of developing future mobility solutions, we need to gain an overall perspective of the energy supply chain. If global warming is to be restricted to a maximum of $2{ }^{\circ} \mathrm{C}$, as enshrined in the Paris climate agreement, it is important for the energy transition to have started in all sectors by 2030. At the colloquium, Schaeffler presented its own scenario. The company believes that a fleet mix of $30 \%$ pure electric, $40 \%$ hybrid, and $30 \%$ conventional powertrains by 2030 is reasonable and feasible. If this scenario is extrapolated to 2050, there would be around $66 \%$ electric vehicles on the road, and the $2{ }^{\circ} \mathrm{C}$ target would be achievable.

The assessment of the mix of powertrains must be based on a cradle-to-grave analysis. "If we look at the big picture, BEVs only have an environmental advantage over PHEVs after 120,000 km, because the environmental footprint of the production process for BEVs is considerable," said Schaeffler Vice President of Research Uwe Wagner, summing up the situation. Because in realistic terms we need shortterm solutions for current powertrains alongside electric systems, the introduction of e-fuels and the hybridization of combustion engines represent important steps toward a lasting global reduction in emissions. If the proportion of e-fuels from sustainable sources increases to $80 \%$ by 2050 as in the Schaeffler scenario, it will be possible to achieve the $\mathrm{CO}_{2}$ targets. But does it then make sense to use one third of the available energy for mobility? There is a lot to be said in favor of a diversified mix of powertrains, including combustion engines and hydrogen. We need to be prepared for a future full of variety.

I hope you enjoy reading this issue of ATZ.

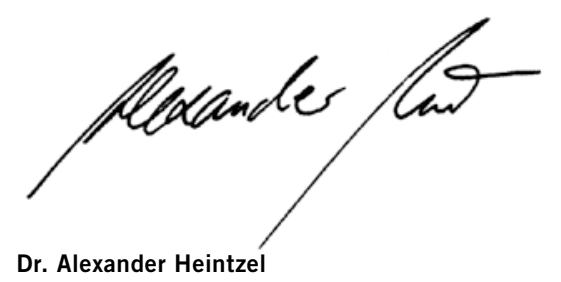
Editor in Chief

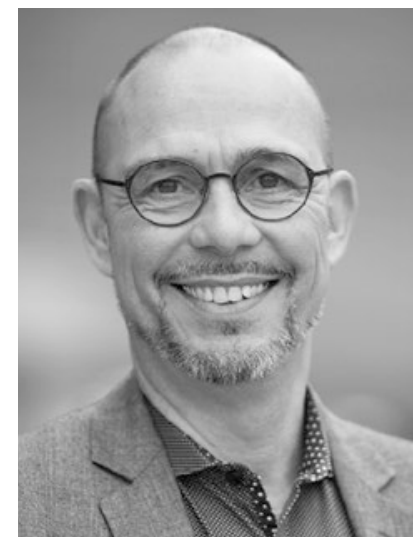

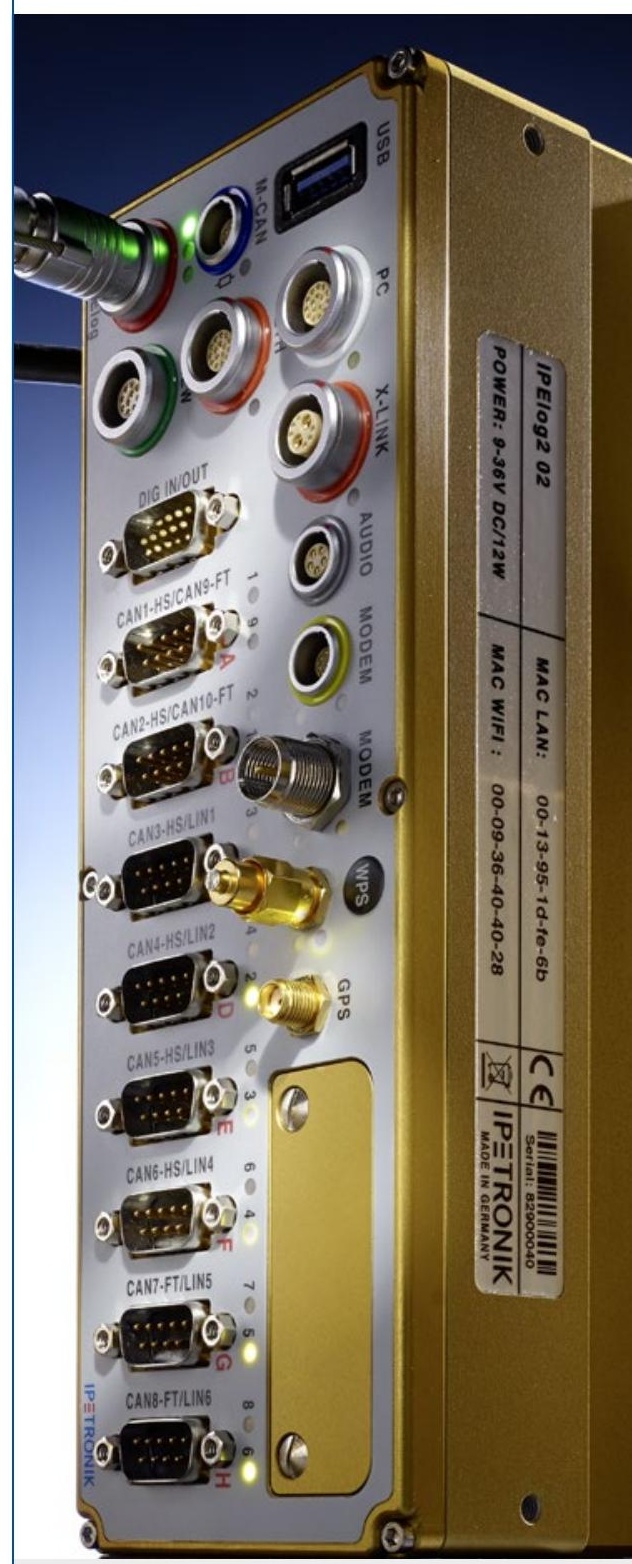

DATALOGGER

FOR THE HIGHEST REQUIREMENTS

- Datalogger for fleet testing and endurance testing worldwide

- Ruggedized hardware for extreme conditions

- Ideally matched hardware and software

- Maximum reliability of data acquisition

- Proven use in hybrid and electric vehicles

- Using latest and innovative technologies 